\title{
Spanning directed trees with many leaves ${ }^{\star}$
}

\author{
Noga Alon ${ }^{1}$, Fedor V. Fomin ${ }^{2}$, Gregory Gutin ${ }^{3}$, Michael Krivelevich ${ }^{1}$, and \\ Saket Saurabh ${ }^{2}$ \\ 1 Department of Mathematics, Tel Aviv University \\ Tel Aviv 69978, Israel \\ \{nogaa, krivelev\}@post.tau.ac.il \\ 2 Department of Informatics, University of Bergen \\ POB 7803, 5020 Bergen, Norway \\ \{fedor.fomin, saket\}@ii.uib.no \\ 3 Department of Computer Science \\ Royal Holloway, University of London \\ Egham, Surrey TW20 0EX, UK \\ gutin@cs.rhul.ac.uk
}

\begin{abstract}
The Directed Maximum Leaf Out-Branching problem is to find an out-branching (i.e. a rooted oriented spanning tree) in a given digraph with the maximum number of leaves. In this paper, we obtain two combinatorial results on the number of leaves in out-branchings. We show that

- every strongly connected $n$-vertex digraph $D$ with minimum indegree at least 3 has an out-branching with at least $(n / 4)^{1 / 3}-1$ leaves;

- if a strongly connected digraph $D$ does not contain an out-branching with $k$ leaves, then the pathwidth of its underlying graph $\mathrm{UG}(D)$ is $O(k \log k)$. Moreover, if the digraph is acyclic, the pathwidth is at most $4 k$.

The last result implies that it can be decided in time $2^{O\left(k \log ^{2} k\right)} \cdot n^{O(1)}$ whether a strongly connected digraph on $n$ vertices has an out-branching with at least $k$ leaves. On acyclic digraphs the running time of our algorithm is $2^{O(k \log k)} \cdot n^{O(1)}$.
\end{abstract}

\section{Introduction}

In this paper, we initiate the combinatorial and algorithmic study of a natural generalization of the well studied MAXimum LeAF Spanning Tree problem on connected undirected graphs $[11,16,19-21,24,25,31,33]$. Given a digraph $D$, a subdigraph $T$ of $D$ is an out-tree if $T$ is an oriented tree with only one vertex $s$ of in-degree zero (called the root). If $T$ is a spanning out-tree, i.e. $V(T)=V(D)$, then $T$ is called an out-branching of $D$. The vertices of $T$ of out-degree zero are called leaves. The Directed Maximum Leaf Out-Branching (DMLOB)

\footnotetext{
* Preliminary extended abstracts of this paper have been presented at FSTTCS 2007

[5] and ICALP 2007 [4]
} 
problem is to find an out-branching in a given digraph with the maximum number of leaves.

The combinatorial study of spanning trees with maximum number of leaves in undirected graphs has an extensive history. Linial conjectured around 1987 that every connected graph on $n$ vertices with minimum vertex degree $\delta$ has a spanning tree with at least $n(\delta-2) /(\delta+1)+c_{\delta}$ leaves, where $c_{\delta}$ depends on $\delta$. This is indeed the case for all $\delta \leq 5$. Kleitman and West [27] and Linial and Sturtevant [30] showed that every connected undirected graph $G$ on $n$ vertices with minimum degree at least 3 has a spanning tree with at least $n / 4+2$ leaves. Griggs and $\mathrm{Wu}[25]$ proved that the maximum number of leaves in a spanning tree is at least $n / 2+2$ when $\delta=5$ and at least $2 n / 5+8 / 5$ when $\delta=4$. All these results are tight. The situation is less clear for $\delta \geq 6$; the first author observed that Linial's conjecture is false for all large values of $\delta$. Indeed, the results in [2] imply that there are undirected graphs with $n$ vertices and minimum degree $\delta$ in which no tree has more than $\left(1-(1+o(1)) \frac{\ln (\delta+1)}{\delta+1}\right) n$ leaves, where the $o(1)$-term tends to zero an $\delta$ tends to infinity, and this is essentially tight. See also [3], pp. $4-5$ and [13] for more information.

In this paper we prove an analogue of the Kleitman-West result for directed graphs: every strongly connected digraph $D$ of order $n$ with minimum in-degree at least 3 has an out-branching with at least $(n / 4)^{1 / 3}-1$ leaves. We do not know whether this bound is tight, however we show that there are strongly connected digraphs with minimum in-degree 3 in which every out-branching has at most $O(\sqrt{n})$ leaves.

Unlike its undirected counterpart which has attracted a lot of attention in all algorithmic paradigms like approximation algorithms [24,31,33], parameterized algorithms [11,19,21], exact exponential time algorithms [20] and also combinatorial studies $[16,25,27,30]$, the Directed Maximum Leaf Out-Branching problem has largely been neglected until recently. The only paper we are aware of is the very recent paper [18] that describes an $O(\sqrt{\mathrm{OPT}})$-approximation algorithms for DMLOB.

Our second combinatorial result relates the number of leaves in a DMLOB of a directed graph $D$ with the pathwidth of its underlying graph $\mathrm{UG}(D)$. (We postpone the definition of pathwidth till the next section.) If an undirected graph $G$ contains a star $K_{1, k}$ as a minor, then it is possible to construct a spanning tree with at least $k$ leaves from this minor. Otherwise, there is no $K_{1, k}$ minor in $G$, and it is possible to prove that the pathwidth of $G$ is $O(k)$. (See, e.g. [8].) Actually, a much more general result due to Bienstock et al. [10]) is that any undirected graph of pathwidth at least $k$, contains all trees on $k$ vertices as a minor. We prove a result that can be viewed as a generalization of known bounds on the number of leaves in a spanning tree of an undirected graph in terms of its pathwidth, to strongly connected digraphs. We show that either a strongly connected digraph $D$ has a DMLOB with at least $k$ leaves or the pathwidth of $\mathrm{UG}(D)$ is $O(k \log k)$. For an acyclic digraph with a DMLOB having $k$ leaves, we prove that the pathwidth is at most $4 k$. This almost matches the bound 
for undirected graphs. These combinatorial results are useful in the design of parameterized algorithms.

In parameterized algorithms, for decision problems with input size $n$, and a parameter $k$, the goal is to design an algorithm with runtime $f(k) n^{O(1)}$, where $f$ is a function of $k$ alone. (For DMLOB such a parameter is the number of leaves in the out-tree.) Problems having such an algorithm are said to be fixed parameter tractable (FPT). The book by Downey and Fellows [17] provides an introduction to the topic of parameterized complexity. For recent developments see the books by Flum and Grohe [23] and by Niedermeier [32].

The parameterized version of DMLOB is defined as follows: Given a digraph $D$ and a positive integral parameter $k$, does $D$ contain an out-branching with at least $k$ leaves? We denote the parameterized versions of DMLOB by $k$-DMLOB. If in the above definition we do not insist on an out-branching and ask whether there exists an out-tree with at least $k$ leaves, we get the parameterized DIRected Maximum Leaf Out-Tree problem (denoted $k$-DMLOT).

Our combinatorial bounds, combined with dynamic programming on graphs of bounded pathwidth imply the first parameterized algorithms for $k$-DMLOB on strongly connected digraphs and acyclic digraphs. We remark that the algorithmic results presented here also hold for all digraphs if we consider $k$-DMLOT rather than $k$-DMLOB. This answers an open question of Mike Fellows [14, $22,26]$. However, we mainly restrict ourselves to $k$-DMLOB for clarity and the harder challenges it poses, and we briefly consider $k$-DMLOT only in the last section.

Very recently, using a modification of our approach, Bonsma and Dorn [12] proved that either an arbitrary digraph $D$ has an out-branching with at most $k$ leaves or the pathwidth of $\mathrm{UG}\left(D^{\prime}\right)$ is $O\left(k^{3}\right)$, where $D^{\prime}$ is the digraph obtained from $D$ by deleting all arcs not contained in any out-branching of $D$. The bound $O\left(k^{3}\right)$ is much larger than our bounds for strongly connected and acyclic digraphs, but it suffices to allow Bonsma and Dorn to show that $k$-DMLOB is FPT, settling another open question of Fellows [22, 26].

This paper is organized as follows. In Section 2 we provide additional terminology and notation as well as some well-known results. We introduce locally optimal out-branchings in Section 3. Bounds on the number of leaves in maximum leaf out-branchings of strongly connected and acyclic digraphs are obtained in Section 4. In Section 5 we prove upper bounds on the pathwidth of the underlying graph of strongly connected and acyclic digraphs that do not contain out-branchings with at least $k$ leaves. In Section 6 we conclude with discussions and open problems.

\section{Preliminaries}

Let $D$ be a digraph. By $V(D)$ and $A(D)$ we represent the vertex set and arc set of $D$, respectively. An oriented graph is a digraph with no directed 2-cycle. Given a subset $V^{\prime} \subseteq V(D)$ of a digraph $D$, let $D\left[V^{\prime}\right]$ denote the digraph induced by $V^{\prime}$. The underlying graph $\operatorname{UG}(D)$ of $D$ is obtained from $D$ by omitting all 
orientations of arcs and by deleting one edge from each resulting pair of parallel edges. The connectivity components of $D$ are the subdigraphs of $D$ induced by the vertices of components of $\mathrm{UG}(D)$. A digraph $D$ is strongly connected if, for every pair $x, y$ of vertices there are directed paths from $x$ to $y$ and from $y$ to $x$. A maximal strongly connected subdigraph of $D$ is called a strong component. A vertex $u$ of $D$ is an in-neighbor (out-neighbor) of a vertex $v$ if $u v \in A(D)$ $\left(v u \in A(D)\right.$, respectively). The in-degree $d^{-}(v)$ (out-degree $\left.d^{+}(v)\right)$ of a vertex $v$ is the number of its in-neighbors (out-neighbors).

We denote by $\ell(D)$ the maximum number of leaves in an out-tree of a digraph $D$ and by $\ell_{s}(D)$ we denote the maximum possible number of leaves in an outbranching of a digraph $D$. When $D$ has no out-branching, we write $\ell_{s}(D)=0$. The following simple result gives necessary and sufficient conditions for a digraph to have an out-branching. This assertion allows us to check whether $\ell_{s}(D)>0$ in time $O(|V(D)|+|A(D)|)$.

Proposition 1 ([7]). A digraph $D$ has an out-branching if and only if $D$ has a unique strong component with no incoming arcs.

Let $P=u_{1} u_{2} \ldots u_{q}$ be a directed path in a digraph $D$. An arc $u_{i} u_{j}$ of $D$ is a forward (backward) arc for $P$ if $i \leq j-2(j<i$, respectively). Every backward arc of the type $v_{i+1} v_{i}$ is called double.

For a natural number $n,[n]$ denotes the set $\{1,2, \ldots, n\}$.

A tree decomposition of an (undirected) graph $G$ is a pair $(X, U)$ where $U$ is a tree whose vertices we will call nodes and $X=\left(\left\{X_{i} \mid i \in V(U)\right\}\right)$ is a collection of subsets of $V(G)$ such that

1. $\bigcup_{i \in V(U)} X_{i}=V(G)$,

2. for each edge $\{v, w\} \in E(G)$, there is an $i \in V(U)$ such that $v, w \in X_{i}$, and

3. for each $v \in V(G)$ the set of nodes $\left\{i \mid v \in X_{i}\right\}$ forms a subtree of $U$.

The width of a tree decomposition $\left(\left\{X_{i} \mid i \in V(U)\right\}, U\right)$ equals $\max _{i \in V(U)}\left\{\left|X_{i}\right|-\right.$ $1\}$. The treewidth of a graph $G$ is the minimum width over all tree decompositions of $G$.

If in the definitions of a tree decomposition and treewidth we restrict $U$ to be a path, then we have the definitions of path decomposition and pathwidth. We use the notation $t w(G)$ and $p w(G)$ to denote the treewidth and the pathwidth of a graph $G$.

We also need an equivalent definition of pathwidth in terms of vertex separators with respect to a linear ordering of the vertices. Let $G$ be a graph and let $\sigma=\left(v_{1}, v_{2}, \ldots, v_{n}\right)$ be an ordering of $V(G)$. For $j \in[n]$ put $V_{j}=\left\{v_{i}: i \in[j]\right\}$ and denote by $\partial V_{j}$ all vertices of $V_{j}$ that have neighbors in $V \backslash V_{j}$. Setting $v s(G, \sigma)=\max _{i \in[n]}\left|\partial V_{i}\right|$, we define the vertex separation of $G$ as

$$
v s(G)=\min \{v s(G, \sigma): \sigma \text { is an ordering of } V(G)\} .
$$

The following assertion is well-known. It follows directly from the results of Kirousis and Papadimitriou [29] on interval width of a graph, see also [28].

Proposition $2([\mathbf{2 8}, \mathbf{2 9}])$. For any graph $G, v s(G)=p w(G)$. 


\section{Locally Optimal Out-Branchings}

Our bounds are based on finding locally optimal out-branchings. Given a digraph, $D$ and an out-branching $T$, we call a vertex leaf, link and branch if its out-degree in $T$ is 0,1 and $\geq 2$ respectively. Let $S_{\geq 2}^{+}(T)$ be the set of branch vertices, $S_{1}^{+}(T)$ the set of link vertices and $L(T)$ the set of leaves in the tree $T$. Let $\mathscr{P}_{2}(T)$ be the set of maximal paths consisting of link vertices. By $p(v)$ we denote the parent of a vertex $v$ in $T ; p(v)$ is the unique in-neighbor of $v$. We call a pair of vertices $u$ and $v$ siblings if they do not belong to the same path from the root $r$ in $T$. We start with the following well known and easy to observe facts.

Fact $1\left|S_{\geq 2}^{+}(T)\right| \leq|L(T)|-1$.

Fact $2\left|\mathscr{P}_{2}(T)\right| \leq 2|L(T)|-1$.

Now we define the notion of local exchange which is intensively used in our proofs.

Definition $3 \ell$-ArC ExChAnge ( $\ell$-AE) OPtimal OUT-BRANCHING: An outbranching $T$ of a directed graph $D$ with $k$ leaves is $\ell-A E$ optimal if for all arc subsets $F \subseteq A(T)$ and $X \subseteq A(D)-A(T)$ of size $\ell,(A(T) \backslash F) \cup X$ is either not an out-branching, or an out-branching with at most $k$ leaves. In other words, $T$ is $\ell-A E$ optimal if it can't be turned into an out-branching with more leaves by exchanging $\ell$ arcs.

Let us remark, that for every fixed $\ell$, an $\ell$-AE optimal out-branching can be obtained in polynomial time. In our proofs we use only 1-AE optimal outbranchings. We need the following simple properties of 1-AE optimal out-branchings.

Lemma 1. Let $T$ be an 1-AE optimal out-branching rooted at $r$ in a digraph $D$. Then the following holds:

(a) For every pair of siblings $u, v \in V(T) \backslash L$ with $d_{T}^{+}(p(v))=1$, there is no arc $e=(u, v) \in A(D) \backslash A(T)$;

(b) For every pair of vertices $u, v \notin L, d_{T}^{+}(p(v))=1$, which are on the same path from the root with $\operatorname{dist}(r, u)<\operatorname{dist}(r, v)$ there is no arc $e=(u, v) \in$ $A(D) \backslash A(T)$ (here $\operatorname{dist}(r, u)$ is the distance to $u$ in $T$ from the root $r$ );

(c) There is no arc $(v, r), v \notin L$ such that the directed cycle formed by the $(r, v)$-path and the arc $(v, r)$ contains a vertex $x$ such that $d_{T}^{+}(p(x))=1$.

Proof. The proof easily follows from the fact that the existence of any of these arcs contradicts the local optimality of $T$ with respect to 1-AE.

\section{Combinatorial Bounds}

We start with a lemma that allows us to obtain lower bounds on $\ell_{s}(D)$. 
Lemma 2. Let $D$ be a oriented graph of order $n$ in which every vertex is of in-degree 2 and let $D$ have an out-branching. If $D$ has no out-tree with $k$ leaves, then $n \leq 4 k^{3}$.

Proof. Let us assume that $D$ has no out-tree with $k$ leaves. Consider an outbranching $T$ of $D$ with $p<k$ leaves which is 1-AE optimal. Let $r$ be the root of $T$.

We will bound the number $n$ of vertices in $T$ as follows. Every vertex of $T$ is either a leaf, or a branch vertex, or a link vertex. By Facts 1 and 2 we already have bounds on the number of leaf and branch vertices as well as the number of maximal paths consisting of link vertices. So to get an upper bound on $n$ in terms of $k$, it suffices to bound the length of each maximal path consisting of link vertices. Let us consider such a path $P$ and let $x, y$ be the first and last vertices of $P$, respectively.

The vertices of $V(T) \backslash V(P)$ can be partitioned into four classes as follows:

(a) ancestor vertices: the vertices which appear before $x$ on the $(r, x)$-path of $T$;

(b) descendant vertices : the vertices appearing after the vertices of $P$ on paths of $T$ starting at $r$ and passing through $y$;

(c) sink vertices: the vertices which are leaves but not descendant vertices;

(d) special vertices: none-of-the-above vertices.

Let $P^{\prime}=P-x$, let $z$ be the out-neighbor of $y$ on $T$ and let $T_{z}$ be the subtree of $T$ rooted at $z$. By Lemma 1, there are no $\operatorname{arcs}$ from special or ancestor vertices to the path $P^{\prime}$. Let $u v$ be an arc of $A(D) \backslash A\left(P^{\prime}\right)$ such that $v \in V\left(P^{\prime}\right)$. There are two possibilities for $u$ : (i) $u \notin V\left(P^{\prime}\right)$, (ii) $u \in V\left(P^{\prime}\right)$ and $u v$ is backward for $P^{\prime}$ (there are no forward arcs for $P^{\prime}$ since $T$ is 1-AE optimal). Note that every vertex of type (i) is either a descendant vertex or a sink. Observe also that the backward arcs for $P^{\prime}$ form a vertex-disjoint collection of out-trees with roots at vertices that are not terminal vertices of backward $\operatorname{arcs}$ for $P^{\prime}$. These roots are terminal vertices of arcs in which first vertices are descendant vertices or sinks.

We denote by $\left\{u_{1}, u_{2}, \ldots, u_{s}\right\}$ and $\left\{v_{1}, v_{2}, \ldots, v_{t}\right\}$ the sets of vertices on $P^{\prime}$ which have in-neighbors that are descendant vertices and sinks, respectively. Let the out-tree formed by backward arcs for $P^{\prime}$ rooted at $w \in\left\{u_{1}, \ldots, u_{s}, v_{1}, \ldots, v_{t}\right\}$ be denoted by $T(w)$ and let $l(w)$ denote the number of leaves in $T(w)$. Observe that the following is an out-tree rooted at $z$ :

$$
T_{z} \cup\left\{\left(i n\left(u_{1}\right), u_{1}\right), \ldots,\left(i n\left(u_{s}\right), u_{s}\right)\right\} \cup \bigcup_{i=1}^{s} T\left(u_{i}\right),
$$

where $\left\{i n\left(u_{1}\right), \ldots, i n\left(u_{s}\right)\right\}$ are the in-neighbors of $\left\{u_{1}, \ldots, u_{s}\right\}$ on $T_{z}$. This outtree has at least $\sum_{i=1}^{s} l\left(u_{i}\right)$ leaves and, thus, $\sum_{i=1}^{s} l\left(u_{i}\right) \leq k-1$. Let us denote the subtree of $T$ rooted at $x$ by $T_{x}$ and let $\left\{i n\left(v_{1}\right), \ldots, i n\left(v_{t}\right)\right\}$ be the in-neighbors of $\left\{v_{1}, \ldots, v_{t}\right\}$ on $T-V\left(T_{x}\right)$. Then we have the following out-tree:

$$
\left(T-V\left(T_{x}\right)\right) \cup\left\{\left(i n\left(v_{1}\right), v_{1}\right), \ldots,\left(i n\left(v_{t}\right), v_{t}\right)\right\} \cup \bigcup_{i=1}^{t} T\left(v_{i}\right)
$$


with at least $\sum_{i=1}^{t} l\left(v_{i}\right)$ leaves. Thus, $\sum_{i=1}^{t} l\left(v_{i}\right) \leq k-1$.

Consider a path $R=v_{0} v_{1} \ldots v_{r}$ formed by backward arcs. Observe that the $\operatorname{arcs}\left\{v_{i} v_{i+1}: 0 \leq i \leq r-1\right\} \cup\left\{v_{j} v_{j}^{+}: 1 \leq j \leq r\right\}$ form an out-tree with $r$ leaves, where $v_{j}^{+}$is the out-neighbor of $v_{j}$ on $P$. Thus, there is no path of backward arcs of length more than $k-1$. Every out-tree $T(w), w \in\left\{u_{1}, \ldots, u_{s}\right\}$ has $l(w)$ leaves and, thus, its arcs can be decomposed into $l(w)$ paths, each of length at most $k-1$. Now we can bound the number of arcs in all the trees $T(w), w \in\left\{u_{1}, \ldots, u_{s}\right\}$, as follows: $\sum_{i=1}^{s} l\left(u_{i}\right)(k-1) \leq(k-1)^{2}$. We can similarly bound the number of arcs in all the trees $T(w), w \in\left\{v_{1}, \ldots, v_{s}\right\}$ by $(k-1)^{2}$. Recall that the vertices of $P^{\prime}$ can be either terminal vertices of backward arcs for $P^{\prime}$ or vertices in $\left\{u_{1}, \ldots, u_{s}, v_{1}, \ldots, v_{t}\right\}$. Observe that $s+t \leq 2(k-1)$ since $\sum_{i=1}^{s} l\left(u_{i}\right) \leq k-1$ and $\sum_{i=1}^{t} l\left(v_{i}\right) \leq k-1$.

Thus, the number of vertices in $P$ is bounded from above by $1+2(k-1)+$ $2(k-1)^{2}$. Therefore,

$$
\begin{aligned}
n & =|L(T)|+\left|S_{\geq 2}^{+}(T)\right|+\left|S_{1}^{+}(T)\right| \\
& =|L(T)|+\left|S_{\geq 2}^{+}(T)\right|+\sum_{P \in \mathscr{P}_{2}(T)}|V(P)| \\
& \leq(k-1)+(k-2)+(2 k-3)\left(2 k^{2}-2 k+1\right) \\
& <4 k^{3} .
\end{aligned}
$$

Thus, we conclude that $n \leq 4 k^{3}$.

Theorem 4. Let $D$ be a strongly connected digraph with $n$ vertices.

(a) If $D$ is an oriented graph with minimum in-degree at least 2 , then $\ell_{s}(D) \geq$ $(n / 4)^{1 / 3}-1$.

(b) If $D$ is a digraph with minimum in-degree at least 3 , then $\ell_{s}(D) \geq(n / 4)^{1 / 3}-$ 1.

Proof. Since $D$ is strongly connected, we have $\ell(D)=\ell_{s}(D)>0$. Let $T$ be an 1-AE optimal out-branching of $D$ with maximum number of leaves. (a) Delete some arcs from $A(D) \backslash A(T)$, if needed, such that the in-degree of each vertex of $D$ becomes 2 . Now the inequality $\ell_{s}(D) \geq(n / 4)^{1 / 3}-1$ follows from Lemma 2 and the fact that $\ell(D)=\ell_{s}(D)$.

(b) Let $P$ be the path formed in the proof of Lemma 2. (Note that $A(P) \subseteq$ $A(T)$.) Delete every double arc of $P$, in case there are any, and delete some more arcs from $A(D) \backslash A(T)$, if needed, to ensure that the in-degree of each vertex of $D$ becomes 2 . It is not difficult to see that the proof of Lemma 2 remains valid for the new digraph $D$. Now the inequality $\ell_{s}(D) \geq(n / 4)^{1 / 3}-1$ follows from Lemma 2 and the fact that $\ell(D)=\ell_{s}(D)$.

Remark 5 It is easy to see that Theorem 4 holds also for acyclic digraphs $D$ with $\ell_{s}(D)>0$.

While we do not know whether the bounds of Theorem 4 are tight, we can show that no linear bounds are possible. The following result is formulated for Part (b) of Theorem 4, but a similar result holds for Part (a) as well. 
Theorem 6. For each $t \geq 6$ there is a strongly connected digraph $H_{t}$ of order $n=t^{2}+1$ with minimum in-degree 3 such that $0<\ell_{s}\left(H_{t}\right)=O(t)$.

Proof. Let $V\left(H_{t}\right)=\{r\} \cup\left\{u_{1}^{i}, u_{2}^{i}, \ldots, u_{t}^{i} \mid i \in[t]\right\}$ and

$$
\begin{aligned}
A\left(H_{t}\right)= & \left\{u_{j}^{i} u_{j+1}^{i}, u_{j+1}^{i} u_{j}^{i} \mid i \in[t], j \in\{0,1, \ldots, t-3\}\right\} \\
& \bigcup\left\{u_{j}^{i} u_{j-2}^{i} \mid i \in[t], j \in\{3,4, \ldots, t-2\}\right\} \\
& \bigcup\left\{u_{j}^{i} u_{q}^{i} \mid i \in[t], t-3 \leq j \neq q \leq t\right\},
\end{aligned}
$$

where $u_{0}^{i}=r$ for every $i \in[t]$. It is easy to check that $0<\ell_{s}\left(H_{t}\right)=O(t)$.

\section{$5 \quad$ Pathwidth of underlying graphs and parameterized algorithms}

By Proposition 1, an acyclic digraph $D$ has an out-branching if and only if $D$ possesses a single vertex of in-degree zero.

Theorem 7. Let $D$ be an acyclic digraph with a single vertex of in-degree zero. Then either $\ell_{s}(D) \geq k$ or the underlying undirected graph of $D$ is of pathwidth at most $4 k$ and we can obtain this path decomposition in polynomial time.

Proof. Assume that $\ell_{s}(D) \leq k-1$. Consider a 1-AE optimal out-branching $T$ of $D$. Notice that $|L(T)| \leq k-1$. Now remove all the leaves and branch vertices from the tree $T$. The remaining vertices form maximal directed paths consisting of link vertices. Delete the first vertices of all paths. As a result we obtain a collection $\mathcal{Q}$ of directed paths. Let $H=\cup_{P \in \mathcal{Q}} P$. We will show that every arc $u v$ with $u, v \in V(H)$ is in $H$.

Let $P^{\prime} \in \mathcal{Q}$. As in the proof of Lemma 2, we see that there are no forward $\operatorname{arcs}$ for $P^{\prime}$. Since $D$ is acyclic, there are no backward $\operatorname{arcs}$ for $P^{\prime}$. Suppose $u v$ is an arc of $D$ such that $u \in R^{\prime}$ and $v \in P^{\prime}$, where $R^{\prime}$ and $P^{\prime}$ are distinct paths from $\mathcal{Q}$. As in the proof of Lemma 2, we see that $u$ is either a sink or a descendent vertex for $P^{\prime}$ in $T$. Since $R^{\prime}$ contains no sinks of $T, u$ is a descendent vertex, which is impossible as $D$ is acyclic. Thus, we have proved that $p w(\mathrm{UG}(H))=1$.

Consider a path decomposition of $H$ of width 1 . We can obtain a path decomposition of $\mathrm{UG}(D)$ by adding all the vertices of $L(T) \cup S_{\geq 2}^{+}(T) \cup F(T)$, where $F(T)$ is the set of first vertices of maximal directed paths consisting of link vertices of $T$, to each of the bags of a path decomposition of $H$ of width 1 . Observe that the pathwidth of this decomposition is bounded from above by

$$
|L(T)|+\left|S_{\geq 2}^{+}(T)\right|+|F(T)|+1 \leq(k-1)+(k-2)+(2 k-3)+1 \leq 4 k-5 .
$$

The bounds on the various sets in the inequality above follows from Facts 1 and 2. This proves the theorem.

Corollary 1. For acyclic digraphs, the problem $k-D M L O B$ can solved in time $2^{O(k \log k)} \cdot n^{O(1)}$. 
Proof. The proof of Theorem 7 can be easily turned into a polynomial time algorithm to either build an out-branching of $D$ with at least $k$ leaves or to show that $p w(\mathrm{UG}(D)) \leq 4 k$ and provide the corresponding path decomposition. A standard dynamic programming over the path (tree) decomposition (see e.g. [6]) gives us an algorithm of running time $2^{O(k \log k)} \cdot n^{O(1)}$.

The following simple lemma is well known, see, e.g., [15].

Lemma 3. Let $T=(V, E)$ be an undirected tree and let $w: V \rightarrow \mathbb{R}^{+} \cup\{0\}$ be a weight function on its vertices. There exists a vertex $v \in T$ such that the weight of every subtree $T^{\prime}$ of $T-v$ is at most $w(T) / 2$, where $w(T)=\sum_{v \in V} w(v)$.

Let $D$ be a strongly connected digraph with $\ell_{s}(D)=\lambda$ and let $T$ be an outbranching of $D$ with $\lambda$ leaves. Consider the following decomposition of $T$ (called a $\beta$-decomposition) which will be useful in the proof of Theorem 8 .

Assign weight 1 to all leaves of $T$ and weight 0 to all non-leaves of $T$. By Lemma $3, T$ has a vertex $v$ such that each component of $T-v$ has at most $\lambda / 2+1$ leaves (if $v$ is not the root and its in-neighbor $v^{-}$in $T$ is a link vertex, then $v^{-}$becomes a new leaf). Let $T_{1}, T_{2}, \ldots, T_{s}$ be the components of $T-v$ and let $l_{1}, l_{2}, \ldots, l_{s}$ be the numbers of leaves in the components. Notice that $\lambda \leq \sum_{i=1}^{s} l_{i} \leq \lambda+1$ (we may get a new leaf). We may assume that $l_{s} \leq l_{s-1} \leq$ $\cdots \leq l_{1} \leq \lambda / 2+1$. Let $j$ be the first index such that $\sum_{i=1}^{j} l_{i} \geq \frac{\lambda}{2}+1$. Consider two cases: (a) $l_{j} \leq(\lambda+2) / 4$ and (b) $l_{j}>(\lambda+2) / 4$. In Case (a), we have

$$
\frac{\lambda+2}{2} \leq \sum_{i=1}^{j} l_{i} \leq \frac{3(\lambda+2)}{4} \text { and } \frac{\lambda-6}{4} \leq \sum_{i=j+1}^{s} l_{i} \leq \frac{\lambda}{2} .
$$

In Case (b), we have $j=2$ and

$$
\frac{\lambda+2}{4} \leq l_{1} \leq \frac{\lambda+2}{2} \text { and } \frac{\lambda-2}{2} \leq \sum_{i=2}^{s} l_{i} \leq \frac{3 \lambda+2}{4} .
$$

Let $p=j$ in Case (a) and $p=1$ in Case (b). Add to $D$ and $T$ a copy $v^{\prime}$ of $v$ (with the same in- and out-neighbors). Then the number of leaves in each of the out-trees

$$
T^{\prime}=T\left[\{v\} \cup\left(\cup_{i=1}^{p} V\left(T_{i}\right)\right)\right] \text { and } T^{\prime \prime}=T\left[\left\{v^{\prime}\right\} \cup\left(\cup_{i=p+1}^{s} V\left(T_{i}\right)\right)\right]
$$

is between $\lambda(1+o(1)) / 4$ and $3 \lambda(1+o(1)) / 4$. Observe that the vertices of $T^{\prime}$ have at most $\lambda+1$ out-neighbors in $T^{\prime \prime}$ and the vertices of $T^{\prime \prime}$ have at most $\lambda+1$ out-neighbors in $T^{\prime}$ (we add 1 to $\lambda$ due to the fact that $v$ 'belongs' to both $T^{\prime}$ and $\left.T^{\prime \prime}\right)$.

Similarly to deriving $T^{\prime}$ and $T^{\prime \prime}$ from $T$, we can obtain two out-trees from $T^{\prime}$ and two out-trees from $T^{\prime \prime}$ in which the numbers of leaves are approximately between a quarter and three quarters of the number of leaves in $T^{\prime}$ and $T^{\prime \prime}$, respectively. Observe that after $O(\log \lambda)$ 'dividing' steps, we will end up with $O(\lambda)$ out-trees with just one leaf, i.e., directed paths. These paths contain $O(\lambda)$ copies of vertices of $D$ (such as $v^{\prime}$ above). After deleting the copies, we obtain a collection of $O(\lambda)$ disjoint directed paths covering $V(D)$. 
Theorem 8. Let $D$ be a strongly connected digraph. Then either $\ell_{s}(D) \geq k$ or the underlying undirected graph of $D$ is of pathwidth $O(k \log k)$.

Proof. We may assume that $\ell_{s}(D)<k$. Let $T$ be be a 1-AE optimal outbranching. Consider a $\beta$-decomposition of $T$. The decomposition process can be viewed as a tree $\mathcal{T}$ rooted in a node (associated with) $T$. The children of $T$ in $\mathcal{T}$ are nodes (associated with) $T^{\prime}$ and $T^{\prime \prime}$; the leaves of $\mathcal{T}$ are the directed paths of the decomposition. The first layer of $\mathcal{T}$ is the node $T$, the second layer are $T^{\prime}$ and $T^{\prime \prime}$, the third layer are the children of $T^{\prime}$ and $T^{\prime \prime}$, etc. In what follows, we do not distinguish between a node $Q$ of $\mathcal{T}$ and the tree associated with the node. Assume that $\mathcal{T}$ has $t$ layers. Notice that the last layer consists of (some) leaves of $\mathcal{T}$ and that $t=O(\log k)$, which was proved above $(k \leq \lambda-1)$.

Let $Q$ be a node of $\mathcal{T}$ at layer $j$. We will prove that

$$
p w(\mathrm{UG}(D[V(Q)]))<2(t-j+2.5) k
$$

Since $t=O(\log k)$, (1) for $j=1$ implies that the underlying undirected graph of $D$ is of pathwidth $O(k \log k)$.

We first prove (1) for $j=t$ when $Q$ is a path from the decomposition. Let $W=\left(L(T) \cup S_{\geq 2}^{+}(T) \cup F(T)\right) \cap V(Q)$, where $F(T)$ is the set of first vertices of maximal paths of $T$ consisting of link vertices. As in the proof of Theorem 7, it follows from Facts 1 and 2 that $|W|<4 k$. Obtain a digraph $R$ by deleting from $D[V(Q)]$ all arcs in which at least one end-vertex is in $W$ and which are not arcs of $Q$. As in the proof of Theorem 7, it follows from Lemma 1 and 1-AE optimality of $T$ that there are no forward $\operatorname{arcs}$ for $Q$ in $R$. Let $Q=v_{1} v_{2} \ldots v_{q}$. For every $j \in[q]$, let $V_{j}=\left\{v_{i}: i \in[j]\right\}$. If for some $j$ the set $V_{j}$ contained $k$ vertices, say $\left\{v_{1}^{\prime}, v_{2}^{\prime}, \cdots, v_{k}^{\prime}\right\}$, having in-neighbors in the set $\left\{v_{j+1}, v_{j+2}, \ldots, v_{q}\right\}$, then $D$ would contain an out-tree with $k$ leaves formed by the path $v_{j+1} v_{j+2} \ldots v_{q}$ together with a backward arc terminating at $v_{i}^{\prime}$ from a vertex on the path for each $1 \leq i \leq k$, a contradiction. Thus $v s\left(\mathrm{UG}\left(D_{2}[P]\right)\right) \leq k$. By Proposition 2 , the pathwidth of $\mathrm{UG}(R)$ is at most $k$. Let $\left(X_{1}, X_{2}, \ldots, X_{s}\right)$ be a path decomposition of $\operatorname{UG}(R)$ of width at most $k$. Then $\left(X_{1} \cup W, X_{2} \cup W, \ldots, X_{s} \cup W\right)$ is a path decomposition of $\mathrm{UG}(D[V(Q)])$ of width less than $k+4 k$. Thus,

$$
p w(\mathrm{UG}(D[V(Q)]))<5 k
$$

Now assume that we have proved (1) for $j=i$ and show it for $j=i-1$. Let $Q$ be a node of layer $i-1$. If $Q$ is a leaf of $\mathcal{T}$, we are done by (2). So, we may assume that $Q$ has children $Q^{\prime}$ and $Q^{\prime \prime}$ which are nodes of layer $i$. In the $\beta$-decomposition of $T$ given before this theorem, we saw that the vertices of $T^{\prime}$ have at most $\lambda+1$ out-neighbors in $T^{\prime \prime}$ and the vertices of $T^{\prime \prime}$ have at most $\lambda+1$ out-neighbors in $T^{\prime}$. Similarly, we can see that (in the $\beta$-decomposition of this proof) the vertices of $Q^{\prime}$ have at most $k$ out-neighbors in $Q^{\prime \prime}$ and the vertices of $Q^{\prime \prime}$ have at most $k$ out-neighbors in $Q^{\prime}$ (since $k \leq \lambda-1$ ). Let $Y$ denote the set of the above-mentioned out-neighbors on $Q^{\prime}$ and $Q^{\prime \prime} ;|Y| \leq 2 k$. Delete from $D\left[V\left(Q^{\prime}\right) \cup V\left(Q^{\prime \prime}\right)\right]$ all arcs in which at least one end-vertex is in $Y$ and which do not belong to $Q^{\prime} \cup Q^{\prime \prime}$ 
Let $G$ denote the obtained digraph. Observe that $G$ is disconnected and $G\left[V\left(Q^{\prime}\right)\right]$ and $G\left[V\left(Q^{\prime \prime}\right)\right]$ are components of $G$. Thus, $p w(\mathrm{UG}(G)) \leq b$, where

$$
b=\max \left\{p w\left(\mathrm{UG}\left(G\left[V\left(Q^{\prime}\right)\right]\right)\right), p w\left(\mathrm{UG}\left(G\left[V\left(Q^{\prime \prime}\right)\right]\right)\right)\right\}<2(t-i+4.5) k
$$

Let $\left(Z_{1}, Z_{2}, \ldots, Z_{r}\right)$ be a path decomposition of $G$ of width at most $b$. Then $\left(Z_{1} \cup Y, Z_{2} \cup Y, \ldots, Z_{r} \cup Y\right)$ is a path decomposition of $\mathrm{UG}\left(D\left[V\left(Q^{\prime}\right) \cup V\left(Q^{\prime \prime}\right)\right]\right)$ of width at most $b+2 k<2(t-i+2.5) k$.

Similar to the proof of Corollary 1, we obtain the following:

Corollary 2. For a strongly connected digraph $D$, the problem $k$-DMLOB can be solved in time $2^{O\left(k \log ^{2} k\right)} \cdot n^{O(1)}$.

\section{Discussion and Open Problems}

In this paper, we initiated the algorithmic and combinatorial study of the DIReCted Maximum Leaf Out-Branching problem. In particular, we showed that for every strongly connected digraph $D$ of order $n$ and with minimum indegree at least $3, \ell_{s}(D)=\Omega\left(n^{1 / 3}\right)$. An interesting open combinatorial question here is whether this bound is tight. If it is not, it would be interesting to find the maximum number $r$ such that $\ell_{s}(D)=\Omega\left(n^{r}\right)$ for every strongly connected digraph $D$ of order $n$ and with minimum in-degree at least 3 . It follows from our results that $\frac{1}{3} \leq r \leq \frac{1}{2}$.

We also provided an algorithm of time complexity $2^{O\left(k \log ^{2} k\right)} \cdot n^{O(1)}$ which solves the $k$-DMLOB problem for a strongly connected digraph $D$. The algorithm is based on a combinatorial bound on the pathwidth of the underlying graph of $D$. Instead of using results from Section 5, one can use Bodlaender's algorithm [9] computing (for fixed $k$ ) tree decomposition of width $k$ (if such a decomposition exists) in linear time. Combined with our combinatorial bounds this yields a linear time algorithm for $k$-DMLOB (for a strongly connected digraphs). However, the exponential dependence of $k$ in Bodlaender's algorithm is $c^{k^{3}}$ for some large constant $c$.

Finally, let us observe that while our results are for strongly connected digraphs, they can be extended to a larger class of digraphs. Notice that $\ell(D) \geq$ $\ell_{s}(D)$ for each digraph $D$. Let $\mathcal{L}$ be the family of digraphs $D$ for which either $\ell_{s}(D)=0$ or $\ell_{s}(D)=\ell(D)$. The following assertion shows that $\mathcal{L}$ includes a large number digraphs including all strongly connected digraphs and acyclic digraphs (and, also, the well-studied classes of semicomplete multipartite digraphs and quasi-transitive digraphs, see [7] for the definitions).

Proposition 3 ([5]). Suppose that a digraph D satisfies the following property: for every pair $R$ and $Q$ of distinct strong components of $D$, if there is an arc from $R$ to $Q$ then each vertex of $Q$ has an in-neighbor in $R$. Then $D \in \mathcal{L}$. 
Let $\mathcal{B}$ be the family of digraphs that contain out-branchings. The results of this paper proved for strongly connected digraphs can be extended to the class $\mathcal{L} \cap \mathcal{B}$ of digraphs since in the proofs we use only the following property of strongly connected digraphs $D: \ell_{s}(D)=\ell(D)>0$.

For a digraph $D$ and a vertex $v$, let $D_{v}$ denote the subdigraph of $D$ induced by all vertices reachable from $v$. Using the $2^{O\left(k \log ^{2} k\right)} \cdot n^{O(1)}$ algorithm for $k$ DMLOB on digraphs in $\mathcal{L} \cap \mathcal{B}$ and the facts that (i) $D_{v} \in \mathcal{L} \cap \mathcal{B}$ for each digraph $D$ and vertex $v$ and (ii) $\ell(D)=\max \left\{\ell_{s}\left(D_{v}\right) \mid v \in V(D)\right\}$ (for details, see [5]), we can obtain an $2^{O\left(k \log ^{2} k\right)} \cdot n^{O(1)}$ algorithm for $k$-DMLOT on all digraphs. For acyclic digraphs, the running time can be reduced to $2^{O(k \log k)} \cdot n^{O(1)}$.

Acknowledgements. Research of N. Alon and M. Krivelevich was supported in part by USA-Israeli BSF grants and by grants from the Israel Science Foundation. Research of F. Fomin was supported in part by the Norwegian Research Council. Research of G. Gutin was supported in part by EPSRC.

\section{References}

1. E. Aarts and J. K. Lenstra, editors. Local search in combinatorial optimization. Wiley-Interscience Series in Discrete Mathematics and Optimization. John Wiley \& Sons Ltd., Chichester, 1997. A Wiley-Interscience Publication.

2. N. Alon, Transversal numbers of uniform hypergraphs. Graphs and Combinatorics 6 (1990), 1-4.

3. N. Alon and J. Spencer, The Probabilistic Method, Second Edition. Wiley, NY, 2000 .

4. N. Alon, F. V. Fomin, G. Gutin, M. Krivelevich, and S. Saurabh, Better Algorithms and Bounds for Directed Maximum Leaf Problems. Lect. Notes Comput. Sci. 4855 (2007), 316-327.

5. N. Alon, F. V. Fomin, G. Gutin, M. Krivelevich and S. Saurabh, Parameterized Algorithms for Directed Maximum Leaf Problems. Lect. Notes Comput. Sci. 4596 (2007), 352-362.

6. S. Arnborg and A. Proskurowski, Linear time algorithms for NP-hard problems restricted to partial $k$-trees, Discrete Appl. Math. 23 (1989), no. 1, 11-24.

7. J. Bang-Jensen and G. Gutin, Digraphs: Theory, Algorithms and Applications. Springer-Verlag, 2000.

8. H.L. Bodlaender, On linear time minor tests and depth-first search. Journal of Algorithms 14 (1993), 1-23.

9. H.L. Bodlaender, A Linear-Time Algorithm for Finding Tree-Decompositions of Small Treewidth. SIAM Journal on Computing 25 (1996), 1305-1317.

10. D. Bienstock, N. Robertson, P. D. Seymour, and R. Thomas. Quickly excluding a forest. J. Comb. Theory Series B, 52:274-283, 1991.

11. P.S. Bonsma, T. Brueggermann and G.J. Woeginger, A faster FPT algorithm for finding spanning trees with many leaves. Lect. Notes Computer Sci. 2747 (2003), 259-268.

12. P.S. Bonsma and F. Dorn, An FPT Algorithm for Directed Spanning k-Leaf. Preprint 046-2007, Combinatorial Optimization \& Graph Algorithms Group, TU Berlin, Nov. 2007.

13. Y. Caro, D. B. West and R. Yuster, Connected domination and spanning trees with many leaves. SIAM J. Discrete Math. 13 (2000), 202-211. 
14. M. Cesati, Compendium of parameterized problems, Sept. 2006. http://bravo.ce.uniroma2.it/home/cesati/research/compendium.pdf

15. F.R.K. Chung, Separator theorems and their applications, In Paths, flows, and VLSI-layout (Bonn, 1988), Series Algorithms Combin., 9 (1990), 17-34, Springer, Berlin.

16. G. Ding, Th. Johnson, and P. Seymour, Spanning trees with many leaves. Journal of Graph Theory 37 (2001), 189-197.

17. R.G. Downey and M.R. Fellows, Parameterized Complexity, Springer-Verlag, 1999.

18. M. Drescher and A. Vetta, An approximation algorithm for the maximum leaf spanning arborescence problem. Manuscript, 2007.

19. V. Estivill-Castro, M.R. Fellows, M.A. Langston, and F.A. Rosamond, FPT is P-Time Extremal Structure I. Proc. ACiD (2005), 1-41.

20. F. V. Fomin, F. Grandoni and D. Kratsch, Solving Connected Dominating Set Faster Than $2^{n}$. Lect. Notes Comput. Sci. 4337 (2006), 152-163.

21. M.R. Fellows, C. McCartin, F.A. Rosamond, and U. Stege, Coordinated kernels and catalytic reductions: An improved FPT algorithm for max leaf spanning tree and other problems. Lect. Notes Comput. Sci. 1974 (2000), 240-251.

22. M. Fellows, Private communications, 2005-2006.

23. J. Flum and M. Grohe, Parameterized Complexity Theory, Springer-Verlag, 2006.

24. G. Galbiati, A. Morzenti, and F. Maffioli, On the approximability of some maximum spanning tree problems. Theoretical Computer Science 181 (1997), 107-118.

25. J.R. Griggs and M. Wu, Spanning trees in graphs of minimum degree four or five. Discrete Mathematics 104 (1992), 167-183.

26. G. Gutin and A. Yeo, Some Parameterized Problems on Digraphs. To appear in The Computer Journal.

27. D.J. Kleitman and D.B. West, Spanning trees with many leaves. SIAM Journal on Discrete Mathematics 4 (1991), 99-106.

28. N. G. Kinnersley, The vertex separation number of a graph equals its path-width, Information Processing Letters 42 (1992), 345-350.

29. L. M. Kirousis and C. H. Papadimitriou, Interval graphs and searching, Discrete Mathematics 55 (1985), 181-184.

30. N. Linial and D. Sturtevant (1987). Unpublished result.

31. H.-I. Lu and R. Ravi, Approximating maximum leaf spanning trees in almost linear time. Journal of Algorithms 29 (1998), 132-141.

32. R. Niedermeier, Invitation to Fixed-Parameter Algorithms, Oxford University Press, 2006.

33. R. Solis-Oba, 2-approximation algorithm for finding a spanning tree with the maximum number of leaves. Lect. Notes Comput. Sci. 1461 (1998), 441-452. 